October $\quad 31$ ffs $2^{\circ} \mathrm{v} 7^{\circ}$ Revised ${ }^{\circ}{ }^{\circ} \mathrm{MPLA}^{\circ}$

Modern Physics Letters A

(C) World Scientific Publishing Company

\title{
ON THE FREQUENCY SHIFT OF GRAVITATIONAL WAVES
}

\author{
CLAUDIO M. G. DE SOUSA \\ Universidade Federal do Para, Faculdade de Fisica Ambiental, \\ Av. Marechal Rondon, s/n, Santarem, PA 68040-070, State of Para, Amazon region, Brazil.* \\ (claudiogomes@ufpa.br, claudio@unb.br) \\ and \\ Universidade Catolica de Brasilia, Diretoria de Fisica e Matematica, \\ QS 07 Lt 01 EPCT Aguas Claras, Brasilia, DF 72030-170, Brazil.
}

Received (Day Month Year)

Revised (Day Month Year)

\begin{abstract}
Considering plane gravitational waves propagating through flat spacetime, it is shown that curvatures experienced both in the starting point and during their arrival at the earth can cause a considerable shift in the frequencies as measured by earth and spacebased detectors. Particularly for the case of resonant bar detectors this shift can cause noise-filters to smother the signal.
\end{abstract}

Keywords: Gravitational waves; geometric optics; frequency shift.

PACS Nos.: 04.30Nk, 95.55.Ym.

\section{Introduction}

The evidence that the decay of a binary pulsar 1 is related to the emission of gravitational waves shows that the predictions of general relativity are valid even if we use approximations as those of the linearized models for small perturbations in the metric tensor.

Despite the claim that gravitational waves have never been directly detected, the study of this subject has afforded large development both in theoretical and experimental fields, not only in general relativity, but also in detectors technology, noise treatment, etc.

Nevertheless, direct detection of gravitational waves can unfold several mysteries of modern cosmology. As an example, the detection of very-long wavelength gravitational waves can improve the knowledge about the origin of the cosmic microwave background 2 . Another example is that of gravitational waves from neutron-starblack-hole spiral that could bound the strength of a scalar field in scalar-tensor theories of gravity ${ }^{3}$, since in those theories there exists the possibility of a dipole

\footnotetext{
*Main address
} 
gravitational radiation. Astrophysical characteristics can also be inferred from the measurements of gravitational waveforms $4 / 5$. It is desirable, thence, that we have a precise idea of gravitational radiation spectra for different sources.

This paper intents to verify the consequences of a frequency shift on gravitational waves that could cause detectors to be working out of their optimum. It is argued here that with few corrections modern detectors will increase their probability of detecting directly gravitational waves.

The foundation of this shift effect is in the Einstein Equivalence Principle 6 (EEP): for instance, if a gravitational wave approaches the earth with a frequency $f$ it could only be detected with the same frequency by a free falling antenna. The inverse problem occurs with gravitational waves outgoing from the source. The influence upon gravitational radiation by its own source is known in the backscattering theory, which involves detailed knowledge of the quadrupole moment of the source 78 . The fact that gravitational waves propagation is similar to electromagnetic waves has been reported by several authors in the backscattering arena 9110 . It is also known the fact that background curvature affects the propagation of the gravitational waves from stochastic cosmological sources 11. Despite backscattering theory has increased with the discussion of a possible suppression of gravitational waves 1213 , and the subsequent demonstration of the absence of such $\omega M$ effects, it is not on the purpose of the present work to show any suppression on gravitational waves, but only the consequences of its frequency deviation to earth based resonant detectors.

In this paper it is also supposed that to quantify the frequency shift effect and to determine if it is worthwhile to be considered, it is not required the full nonlinear theory, and one can extend the geometric optics to regions where the background influences strongly the gravitational radiation. Considering wavelengths short compared to the radius of curvature of the background space-time, one can observe that the propagation equations for the gravitational radiation yields similar phenomena as those observed for the electromagnetic radiation 14 .

Using the metric:

$$
g_{\mu \nu}=\eta_{\mu \nu}+h_{\mu \nu}
$$

where $h_{\mu \nu}$ represents a small perturbation on the flat spacetime metric $\eta_{\mu \nu}$ (with $\left.\left\|h_{\mu \nu}\right\| \ll 1\right)$, the linearized vacuum field equations take the form:

$$
\square h_{\mu \nu}+h_{, \mu \nu}-h_{\nu, \mu \alpha}^{\alpha}-h_{\mu, \nu \alpha}^{\alpha}=0 .
$$

But, with the choice of the gauge 15 .

$$
h_{\mu, \alpha}^{\alpha}-\frac{1}{2} h,_{\mu}=0,
$$

where $h=\eta^{\mu \nu} h_{\mu \nu}$, one obtains:

$$
\square h_{\mu \nu}=0 .
$$


As first attempt to compute the effects of the gravitational field on the gravitational wave in the very moment it comes out from the wave generation zone close to the source, the plane wave formalism will be extended even to regions where the field is strong.

Hence, consider the gravitational radiation propagating as a plane wave given by some solution of the equation (2), in an eikonal type form:

$$
h_{\mu \nu}=\operatorname{Re}\left\{A_{\mu \nu} e^{i k x}\right\},
$$

where $k \cdot x=k_{0} x^{0}+k_{i} x^{i}=\Psi$ (with $i=1,2,3$ ) and $\Psi$ is the eikonal from which the wave vector $\mathrm{k}=-\nabla \Psi$ and the frequency $\omega=\Psi,_{0}$ (i.e., $k_{\mu} \equiv \Psi, \mu$ ) can be obtained.

Considering $A_{\mu \nu}^{*}$ as the complex conjugate of $A_{\mu \nu}$, one can also define the scalar amplitude $A=\sqrt{\frac{1}{2} A_{\mu \nu}^{*} A_{\mu \nu}}$ and the polarization $e_{\mu \nu}=\frac{A_{\mu \nu}}{A}$.

Using the above defined quantities one can develop all geometrical optics formalism for the gravitational waves 16 . The analogy between gravitational waves and light using the eikonal (WKB approximation) was addressed by Isaacson 7 . This approximation becomes more accurate for high-frequencies, when the wavelength is small compared to the radius of curvature of the background geometry, and the Riemann and the Ricci tensors expansions are gauge invariants to an extremely good approximation, since geometry can be considered as locally flat over distances of order $L$, where in first approximation one gets $R_{\mu \nu \alpha \beta}^{(0)} \sim L^{-2}$. Thus considering $\lambda \ll L$ one ensures that $A_{\mu \nu}$ and $k_{\mu}$ vary slowly over a characteristic distance of order $L$.

But the main feature to be considered here is the propagation frequency of gravitational waves in such way that, in the next section, we can analyze the effects of the source on its own gravitational wave frequency.

Noticing that the proper-time is $17 / 18$.

$$
t=\int \sqrt{g_{00}} d x^{0},
$$

we have:

$$
\frac{\partial}{\partial t} h_{\mu \nu}=\frac{\partial h_{\mu \nu}}{\partial x^{0}} \frac{\partial x^{0}}{\partial t}=h_{\mu \nu, 0} \frac{1}{\sqrt{g_{00}}}
$$

and so:

$$
\omega=\frac{\omega_{0}}{\sqrt{g_{00}}}
$$

Since $g_{00}=1+2 \phi / c^{2}$ (recovering natural units, where $c$ is the velocity of light), where $\phi<0$ is the gravitational potential (so normalized that $\phi(\infty)=0$ ), in the linearized theory one can write the approximation:

$$
\omega=\omega_{0}\left(1-\frac{\phi}{c^{2}}\right) .
$$


If the gravitational radiation is emitted (Fig 1) from a point where the potential is $\phi_{1}$ with frequency $\omega$, and is received at a point where the potential is $\phi_{2}$, then the frequency is shifted from its original value in $\Delta \omega$, given by:

$$
\Delta \omega=\left(\frac{\phi_{1}-\phi_{2}}{c^{2}}\right) \omega
$$

which is the first-order Doppler effect formula usually found in electromagnetic redshift investigation. Hence, gravitational waves experience a Doppler-like effect due to the difference between the source and the earth gravitational potentials (neglecting relative velocities).

For instance, taking $\phi_{1}$ as the gravitational potential of the source and $\phi_{2}$ on the earth, most of the detectable events will happen with $\left|\phi_{1}\right| \gg\left|\phi_{2}\right|$ and, hence, $\Delta \omega<$ 0 , showing that there is an overall redshift on gravitational waves to be detected on the earth (except for background gravitational waves that baths the space). Thus, during its travel from the source to a ground based detector gravitational waves will usually be found in a lower frequency than that expected by standard calculations.

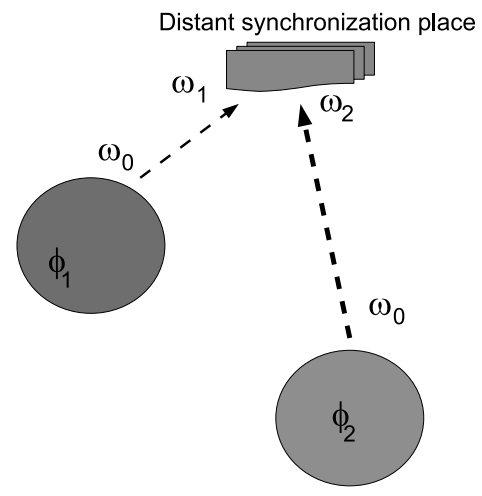

Fig. 1. Scheme used to obtain equation (6) showing the frequency synchronization of two different gravitational potentials, $\phi_{1}$ and $\phi_{2}$. Similar experiments are carried out on the planets emitting signals with the same frequency $\omega_{0}$, which are influenced by the gravitational potentials. This experiment allows one to calibrate $\Delta \omega$.

\section{Sample Calculations}

Some of the expected events in the search of gravitational radiation can be selected to determine if corrections are demanded for modern detectors. 
For the purpose of a simple analysis one can use equation (6) to write:

$$
\frac{\Delta f}{f}=\frac{\phi_{1}-\phi_{2}}{c^{2}}
$$

where $f$ is the source frequency, which has potential $\phi_{1}$.

Typical earth mass and radius give rise to $\phi_{2} \simeq-6 \times 10^{7} \mathrm{~J} / \mathrm{kg}$, negligible compared to the usual gravitational potential for the target sources.

As two examples, consider the cases of a collapsing neutron star and of a black hole. If one considers a collapsing neutron star with $M_{\mathrm{NS}} \simeq M_{\odot}$ and $R_{\mathrm{NS}} \simeq 10^{4} \mathrm{~m}$, and so $\phi_{1}=\phi_{\mathrm{NS}} \simeq-1.3 \times 10^{16} \mathrm{~J} / \mathrm{kg}$, the range 19 of characteristic frequencies is $10^{3} \leq f \leq 10^{4} \mathrm{~Hz}$. Another example to be considered is that of black holes, which have mass $2 M_{\odot} \leq M_{\mathrm{BH}} \leq 10^{10} M_{\odot}$ and, considering the horizon $R_{\mathrm{BH}} \simeq 2 M_{\mathrm{BH}}$, the exact mass of the black hole does not affect the final result for the potential which is always $\phi_{\mathrm{BH}} \approx-G / 2$.

Now, computing the radiation shift for collapsing neutron star case with the use of equation (7), one obtain:

$$
\Delta f_{\mathrm{NS}} \simeq-\frac{\phi_{\mathrm{NS}}}{c^{2}} f_{0}
$$

where $f_{0}$ is the original source frequency. Hence, the gravitational radiation for the collapsing neutron star suggested above gives:

$$
\frac{f_{\mathrm{NS}}}{f_{0}} \simeq 0.8517
$$

and the frequency range is no longer $10^{3} \leq f \leq 10^{4} \mathrm{~Hz}$, but $852 \leq f \leq 8517 \mathrm{~Hz}$.

As can be expected, the effect is stronger the higher is the gravitational potential of the source. For black holes $\Delta f \simeq-\frac{1}{2} f_{0}$, or:

$$
f_{\mathrm{BH}}=\frac{f_{0}}{2}
$$

Some known results 20 are presented on Table 1 with their redshift values predicted by this effect, considering the shifts are caused only by geometries of the source and of the earth. For the compact binary on the Table 1 the mass is approximately $M_{\odot}$ and radius $R_{\odot}$, and the expected redshift is negligible, approximately $10^{-6}$. The stochastic gravitational wave background presents a negligible blueshift for earth-based detectors. Furthermore, the characteristic frequencies for the stochastic GW background $\left(10^{-5}\right.$ to $\left.0.03 \mathrm{~Hz}\right)$ is in the low frequency regime, where the approximations considered here fail. The redshifts have been computed using $1+z=f_{e m} / f_{o b}$, with emitted and observed frequencies, $f_{e m}$ and $f_{o b}$, respectively.

\section{Resonant detectors}

Gravitational waves can experience almost all the characteristics of electromagnetic waves, apart from the properties related to the charges that originated the wave. 
Table 1. Approximate frequency of some sample sources usually considered as targets for gravitational waves search and the expected shifts caused by the gravitational field of the source and of the earth. The gravitational potential for the black hole is in geometrized units. Note that for the example given for coalescence of compact binaries the redshift is negligible. For the stochastic background there is a negligible blueshift.

\begin{tabular}{|l|c|c|c|c|c|}
\hline $\begin{array}{l}\text { Sample } \\
\text { Description }\end{array}$ & $\begin{array}{c}\text { Source Grav. } \\
\text { Potent.(J/kg) }\end{array}$ & $\begin{array}{c}\text { Emitted } \\
\text { Freq. (Hz) }\end{array}$ & $\begin{array}{c}\text { Expected } \\
\text { Freq. }(\mathrm{Hz})\end{array}$ & $\begin{array}{c}\text { Shift } \\
(\mathrm{Hz})\end{array}$ & $\mathrm{z}$ \\
\hline \hline $\begin{array}{l}\text { Collapsing } \\
\text { Neutron Star }\end{array}$ & $-1.3 \times 10^{16}$ & $10^{4}$ & $8.5 \times 10^{3}$ & $14.8 \%$ & 0.1741 \\
\hline $\begin{array}{l}\text { Collapsing } \\
\text { Black Hole }\end{array}$ & $-G / 2$ & $10^{6}$ & $5 \times 10^{5}$ & $50.0 \%$ & 1 \\
\hline $\begin{array}{l}\text { Coalescence } \\
\text { of Compact } \\
\text { Binaries }\end{array}$ & $-5.34 \times 10^{11}$ & $10^{2}$ & $\simeq 10^{2}$ & $\simeq 0.0$ & $\simeq 0$ \\
\hline $\begin{array}{l}\text { Stochastic } \\
\text { Background }\end{array}$ & - & $10^{-5}-0.03$ & $\begin{array}{c}\text { nearly } \\
\text { unchanged }\end{array}$ & $\simeq 0.0$ & $\simeq 0$ \\
\hline
\end{tabular}

Together with those characteristics is the shift of gravitational wave frequency, which is a Doppler-like effect for gravitational waves. But if gravitational waves have their frequency shifted, what could be the consequences for detectors?

Focusing on resonant detectors, which are known as narrow-band around the frequency $f_{0}$ they are looking for, the signal-to-noise ratio is given by 20 .

$$
\frac{S^{2}}{N^{2}}=\frac{\frac{\pi}{2} f_{0}^{2}\left|\tilde{h}\left(f_{0}\right)\right|^{2}}{k T_{n}} \int \sigma_{0}(f) d f
$$

where $\tilde{h}\left(f_{0}\right)$ is the Fourier transform of $h(t), T_{n}$ is the noise temperature (which takes care of all possible noises), and $\sigma_{0}$ is the cross section of the detector. Typically the noise is so severe that it becomes necessary to use a bandwidth $\Delta f$ very small compared to the sought frequency $f_{0}$. Hence, if one considers the case of the black holes in equation (10), the original signal-to-noise ratio $(S N R)_{0}$ can roughly reach:

$$
S N R \simeq \frac{1}{4}(S N R)_{0} .
$$

Neglecting further effects of the $50 \%$ shift on $\tilde{h}\left(f_{0}\right)$, the noise smothers source signal. To better understand the device behaviour with a biased signal, consider resonant detectors as EXPLORER and NAUTILUS. With $S N R=1$, the gravitational wave spectrum is given by 21 :

$$
S_{h}(\omega)=\pi^{2} \frac{k T_{e}}{M Q v^{2}} \frac{\omega_{0}^{3}}{\omega^{4}}\left(1+\Gamma\left(Q^{2}\left(1-\frac{\omega^{2}}{\omega_{0}^{2}}\right)^{2} \frac{\omega^{2}}{\omega_{0}^{2}}\right)\right)
$$

where $k$ is the Boltzmann constant, $T_{e}$ is the equivalent temperature which includes backreaction for the electronic amplifier, $M$ is the cylinder mass, $Q$ is the quality factor of the bar material, $v$ is the sound velocity in the bar material, $\Gamma$ is a characteristic dimensionless factor (usually very small), $\omega$ is the incident frequency and $\omega_{0}$ is the bar natural resonance frequency. 
For $\omega=\omega_{0}$ the detector has its highest sensitivity:

$$
S_{h}\left(\omega_{0}\right)=\pi^{2} \frac{k T_{e}}{M Q v^{2}} \frac{\omega_{0}^{3}}{\omega^{4}}\left(\frac{1+\Gamma}{\omega_{0}}\right)
$$

The spectral amplitude is given by:

$$
\tilde{h}=\sqrt{S_{h}}
$$

For EXPLORER and NAUTILUS one can use $T_{e}=0.1 \mathrm{~K}, M=2270 \mathrm{~kg}, Q=$ $8.5 \times 10^{5}, v=5400 \mathrm{~m} / \mathrm{s}$ (sound velocity in aluminum) and $\Gamma=10^{-6}$.

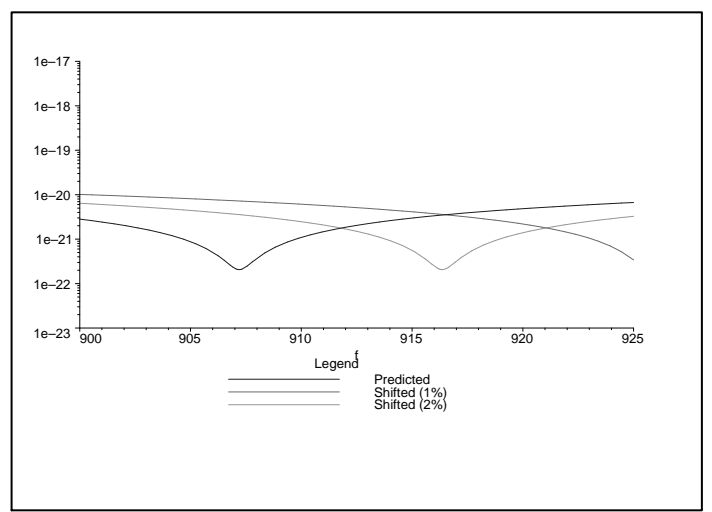

Fig. 2. Spectral amplitudes $\tilde{h}$ versus source frequency. Thick line represents the predicted curve using $f=f_{0}$, with minimum at $907.2 \mathrm{~Hz}$. The minimum of the curve corresponds to maximum sensitivity, and is of order $10^{-22}$, as expected for Explorer and Nautilus. When very small shifts are introduced the minimum is displaced (thin lines). .

Figure 2 shows plots for spectral amplitudes $\tilde{h}$ using eq.(12). The thick line corresponds to the predicted spectrum when the wave reaches detector with the same frequency $\omega$ as that of the bar resonance $\omega_{0}$ (Figure 2 used $f=f_{0}=907.2 \mathrm{~Hz}$ ). The minimum corresponds to expected point of maximum sensitivity of the device, and is of order $10^{-22} \mathrm{~Hz}^{-1 / 2}$. Notice that for slightly shifted values of gravitational waves frequency major modifications are observed on the point of maximum sensitivity.

Figure 3 represents the same scenario for the samples collapsing neutron star $(z=0.17)$ and collapsing black hole $(z=1)$. The sensitivity is of order $10^{-20} \mathrm{~Hz}^{-1 / 2}$ and $10^{-19} \mathrm{~Hz}^{-1 / 2}$, respectively. Nevertheless, this does not mean that detectors are unable to receive signals from sources with different frequencies. It is just matter of time receiving the signal that correctly matches with the sensitivity. However, such frequency shift can seriously decreases the probability of detecting most of the high-intensity sources in the range devices has been projected to.

Similar calculations can be carried out to verify what are the consequences of this effect for broad-band detectors, as GEO600, VIRGO, LIGO and TAMA, which operate as laser interferometric detectors at room temperature, and thermal and 


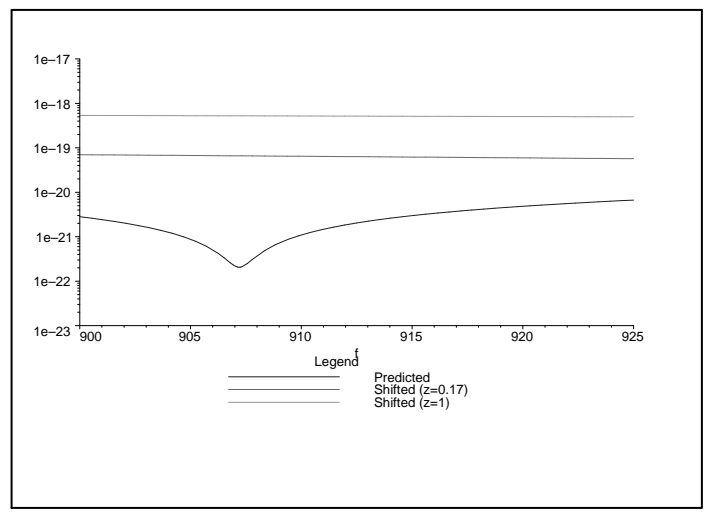

Fig. 3. Spectral amplitudes $\tilde{h}$ versus source frequency for the samples collapsing neutron star $(z=0.17)$ and collapsing black hole $(z=1)$. Thick line represents the predicted curve using $f=f_{0}$. The upper line corresponds to case $z=1$ and the middle line corresponds to case $z=0.17$. In Explorer and Nautilus operating frequency range, there is a huge change in the sensitivity for some of the most expected events.

shot noise become larger compared to the signal 22 , since the power output is proportional to $f^{1 / 2}$.

Besides the highly sophisticated noise treatment 23 necessary to analyze GW detector output, one must extract the filtered signal after considering many factors. For instance, orbital motions of the LISA detector will cause the appearance of a frequency modulation 24 , which can be used to determine the source location and orientation. LISA will work in the low-frequency band, $10^{-4}-10^{-1} \mathrm{~Hz}$ (which, due to seismic noise, is not reached by the ground-based detectors). On the other hand, in LIGO detectors 25 the filtering of seismic noise is so heavy that it creates a cutoff frequency around $10 \mathrm{~Hz}$ at the test masses, in such that some events predicted to fall nearby that cutoff frequency can be missed.

\section{Limits of Validity}

The fact that EEP has been mentioned does not impose the use of a reference frame with no curvature. An event detected by an antenna is considered as an experiment. Due to EEP, an experiment in a free falling laboratory will give same result as if it were in empty space. The fact that the spacetime is locally flat does not contradict with the possibility of small perturbations to propagate, if ripples influence on the background curvature is considered negligible (which is usual for high frequency waves 14 .

Geometric optics 29 requires a large $\Psi$ and that $\lambda \ll \ell(x)$, which is to say the (reduced) wavelength must be very small compared to the radius of curvature of local background. Rewriting (11) in the form $g_{\mu \nu}=g_{\mu \nu}^{\mathrm{B}}+h_{\mu \nu}$. The propagation 14 is due to $h_{\mu \nu}$ and after skipping the generating zone, it is considered that the 
waves reach empty space. For high-frequencies the influence of the waves on $g_{\mu \nu}^{\mathrm{B}}$ is considered negligible. Hence, expressions (5)-(6) consider the background influence on gravitational waves only in the generating zone, and use flat background for the propagation zone.

Discussing further the analysis carried out by Mashhoon 29 concerning the propagation of electromagnetic waves using geometrical optics on a curved manifold, some remarks become necessary in such to ensure the validity of the present geometric optics approximation analogy for gravitational waves:

(i) Two observers moving apart each other induce a frequency Doppler shift

$$
\lambda^{\prime}(x)=\sqrt{\frac{1+\beta}{1-\beta}} \lambda(x)
$$

where $\beta \geq 0$ is the $x$-axis velocity of the observer $\mathcal{O}^{\prime}$ with respect to a stationary observer $\mathcal{O}$ at the origin (i.e., $\beta=v / c$, and $c=1$ in geometrized units). Planets in Fig 1 are imagined to have $\beta \rightarrow 0$, in such that the standard Doppler effect can be neglected;

(ii) Mashhoon's work shows the validity limits of the $\lambda \ll \ell(x)$ assumption in two key cases: exterior Schwarzschild geometry, and the Friedmann-LamaitreRobertson-Walker model universe. A possible conflict appears if $\beta \rightarrow 1$, which can be circumvented using the geometric optics limit, and is here ensured by considering $\beta \rightarrow 0$.

(iii) Finally, one must consider the restrictions on the frequency that can be imposed by the quantum limit for the propagation. Since the source gravitational potential is of order $\phi \sim G \hbar \omega / \lambda \sim\left(L_{P} / \lambda\right)^{2}$, where $L_{P}$ is the Plank length, the influence of the background on the field can be neglected if $\phi \ll 1$ (in geometric units). To ensure this quantum limit does not affect the background for the propagation of the gravitational waves, it is necessary to consider wavelengths such that $\lambda \gg L_{P}$, which corresponds to an upper limit for the frequency, $f \ll 10^{43} \mathrm{~Hz}$. Nevertheless, if $\lambda \sim L_{P}$, quantum effects become considerable.

Despite Mashhoon have evaluated calculations for electromagnetic waves, there is a natural extension for gravitational waves preserving the covariant formalism, and the same limits apply.

\section{Conclusion}

Using the so-called geometrical optics for gravitational waves it is argued that the difference between the gravitational potentials of the source and the earth can cause shifts on gravitational radiation frequencies, which are sometimes considerable. To quantify the consequences of this effect geometric optics formalism has been extended to regions where the background curvature is strong. In the first order approximation carried out here, one can see that very strong sources as black holes can have their frequency shifted to $50 \%$ of the original expected. For some kinds of detectors this shift can cause noise-filters to smother the signal. 
The resulting shift on gravitational waves frequency does not reduce the importance of the presently existing devices. Moreover, it is possible to increase their probability to detect directly gravitational waves bursts if an adequate settlement of experiments take into account the displacement of the original frequency expected. It just emerges from this that another Doppler modulation factor should be taken into account during detector pattern analysis.

Recently, it has been reported 26 that two cryogenic resonant detectors 27 displayed coincident burst events in 1998 and in 2001: EXPLORER operates at frequencies ranging from $904.7 \mathrm{~Hz}$ to $921.3 \mathrm{~Hz}$ and at a thermodynamic temperature of $2.6 \mathrm{~K}$; NAUTILUS operates at $906.97 \mathrm{~Hz}$ to $922.46 \mathrm{~Hz}$ and at a temperature of 1.5K. In 2001, these detectors have obtained six coincidences in five days, approximately one coincidence per day, with an event signal corresponding to a burst with amplitude $h \simeq 2 \times 10^{-18}$. The correlation coefficient is 0.96 , detectors are $600 \mathrm{~km}$ apart and, the connection with present paper is that during those runnings no energy filter was applied. In the reports concerning data obtained from EXPLORER and NAUTILUS, the group preferred to take a conservative position and testing the possibility of the events being result of cosmic ray interference or the possibility of accidental coincidences. The events are reported to be located in the direction of the center of the Galaxy, the same place Weber 28 claimed to be the origin of signals detected in the seventies. The correct choice of the filters (and even the lack of them) could have improved EXPLORER and NAUTILUS detection sensitivity to adjacent frequencies.

\section{Acknowledgments}

I would like to thank L. Ph. Vasconcelos, H. Nazareno, A. Adib and R. Howell for reading the manuscript, and Department of Physics and Astronomy, Dartmouth College, for the hospitality during the initial development of this work. I have also greatly benefited from stimulating discussions with M. D. Maia. This work has been supported by CNPq (Conselho Nacional de Desenvolvimento Cientifico e Tecnologico), Brazil, under the contract 201031/96-5.

\section{References}

1. J. H. Taylor, L. A. Fowler and P. M. McCalloch, Nature 277, 436 (1978); R. A. Hulse and J. H. Taylor, Astrophys. J. 195, L51-L53 (1975).

2. R. R. Caldwell, M. Kamionkowski and L. Wadley, Phys. Rev. D 59, 027101 (1999).

3. P. D. Scharre and C. M. Will, Phys. Rev. D 65, 042002 (2002).

4. M. Vallisneri, Phys. Rev. Lett. 84, 3519 (2000).

5. M. Saijo and T. Nakamura, Phys. Rev. Lett. 85, 2665 (2000).

6. C. M. Will. Theory and Experiment in Gravitational Physics. Cambridge University Press, 1993.

7. R. A. Isaacson, Phys. Rev. 166, 1263 (1968).

8. K. S. Thorne, Rev. Mod. Phys. 52, 299-339 (1980); see also ibid., pp. 285-297 (1980).

9. G. A. Campbell and, R. A. Matzner, J. Math. Phys.. 14, 1 (1973). 
October $\quad 31$ fs $2^{\circ}{ }^{\circ} 7{ }^{\circ}$ Revised ${ }^{\circ}{ }^{\circ} \mathrm{MPLA}$

10. E. Malec and G. Schäfer, Phys. Rev. D 64, 044012 (2001).

11. C. Caprini, R. Durrer and R. Sturani, Phys. Rev. D 74, 127501 (2006).

12. R. H. Price and J. Pullin, Phys. Rev. D 46, 2497 (1992).

13. R. H. Price, J. Pullin and P. K. Kundu, Phys. Rev. Lett. 11, 1572 (1993).

14. C. W. Misner, K. S. Thorne, and, J. A. Wheeler, Gravitation (Freeman, 1973).

15. B. Schutz and F. Ricci, in Gravitational Waves edited by I. Ciufolini et al (IOP, 2001).

16. K. S. Thorne, in Topics in Theoretical and Experimental Gravitational Physics, edited by. V. Sabbata and J. Weber (Plenum Press, 1977).

17. A. Einstein, Annalen der Physik 35, 898 (1911).

18. L. Landau, E. Lifshitz. Classical Theory of Fields. 4. ed. London: Pergamon Press, 1985.

19. E. Mueller, Astronom. Astroph. 114, 53 (1982).

20. K. S. Thorne, in Three Hundred Years of Gravitation, edited by S. W. Hawking and W. Israel (Cambridge U. P., 1987).

21. G. Pizzela, in Gravitational Waves edited by I. Ciufolini et al (IOP, 2001).

22. A. Di Virgilio, in Gravitational Waves edited by I. Ciufolini et al (IOP, 2001).

23. B. F. Schutz, gr-qc/9710080

24. N. J. Cornish and S. L. Larson, Class. Quant. Grav. 20, S163-S170 (2003).

25. P. Fritschel, in Astrophysical Sources for Ground-Based Gravitational Wave Detectors, edited by J. M. Centrella (AIP, 2001).

26. A. Rocchi, in Proceedings of the Tenth Marcel Grossmann Meeting, Rio de Janeiro 2003, pt. C* 1969-1978. Edited by M. Novello, S. Perez Bergliaffa and R. Ruffini. (Hackensack, World Scientific, 2005).

27. P. Astone et al, Class. Quant. Grav. 18, 243 (2001); P. Astone et al, Class. Quant. Grav. 18, 5449 (2002)

28. J. Weber, Phys. Rev. Lett. 25, 180 (1970).

29. B. Mashhoon, Phys. Lett. A122, 299-304 (1986). 